\title{
Criminologie
}

\section{Femmes et héroïne : bilan des connaissances, limites et perspectives nouvelles}

\section{Sylvie Frigon}

Volume 22, numéro 1, 1989

Drogues et criminalités

URI : https://id.erudit.org/iderudit/017275ar

DOI : https://doi.org/10.7202/017275ar

Aller au sommaire du numéro

\section{Éditeur(s)}

Les Presses de l'Université de Montréal

ISSN

0316-0041 (imprimé)

1492-1367 (numérique)

Découvrir la revue

Citer cet article

Frigon, S. (1989). Femmes et héroïne : bilan des connaissances, limites et perspectives nouvelles. Criminologie, 22(1), 85-109.

https://doi.org/10.7202/017275ar
Résumé de l'article

This article gives the results of the consultation of several bases of bibliographical data on Women and Heroin, using the Boolean type of research. Various subjects are discussed, the extent of its use, the characteristics and factors of involvement in drug dependency, treatment, Mother/children relationship, Aids and prostitution. In the light of this survey of the literature, it seemed necessary to question the actual pertinence of these studies. They pose a number of important problems. Our criticism of the quality of these studies are I) problems of method and sampling; 2) epistemological problems and 3) the "monodisciplinarity" of these studies. In these works, the norm or "normality" (of drug addiction) is proper to the male sex. Women are considered marginal to this norm. Other fundamental omissions are mentioned, especially the relativity of the norms, problems connected with the concepts and the terms used. With regard to these various criticisms, we propose two models of analysis. The first refers to the study of women heroin-users as a group rather than in regard to men. The second is to construct a more general plan of analysis ofmarginality, of deviance and the criminality of women using the theories of social reaction in criminology and of radical materialist feminism. These two models could be complementary. 


\section{FEMMES ET HÉROIINNE: BILAN DES CONNAISSANCES,}

LIMITES ET PERSPECTIVES NOUVELLES

Sylvie Frigon*

This article gives the results of the consultation of several bases of bibliographical data on Women and Heroin, using the Boolean type of research. Various subjects are discussed, the extent of its use, the characteristics and factors of involvement in drug dependency, treatment, Mother/children relationship, Aids and prostitution. In the light of this survey of the literature, it seemed necessary to question the actual pertinence of these studies. They pose a number of important problems. Our criticism of the quality of these studies are 1) problems of method and sampling; 2) epistemological problems and 3) the "monodisciplinarity" of these studies. In these works, the norm or "normality" (of drug addiction) is proper to the male sex. Women are considered marginal to this norm. Other fundamental omissions are mentioned, especially the relativity of the norms, problems connected with the concepts and the terms used. With regard to these various criticisms, we propose two models of analysis. The first refers to the study of women heroin-users as a group rather than in regard to men. The second is to construct a more general plan of analysis of marginality, of deviance and the criminality of women using the theories of social reaction in criminology and of radical materialist feminism. These two models could be complementary.

Cet article présente une facette particulière de la «déviance des femmes»; celle qui s'exprime en relation de la consommation d'héroïne. Cette déviance est postulée par plusieurs auteurs qui se penchent sur la consommation d'héroïne mais davantage lorsqu'il est question de femmes.

Dans un premier temps, nous présenterons notre problématique et notre méthode; dans un deuxième temps, nous ferons état des connaissances sur les femmes consommatrices d'héroïne; et, dans un troisième temps, nous entreprendrons l'interprétation critique des résultats et avancerons quelques pistes pertinentes de recherche.

* L'auteure reprend ici l'analyse de son mémoire de maîtrise «Les femmes et l'héroüne: bilan des connaissances et perspectives de recherche», École de criminologie, Université de Montréal, 1987. Elle est présentement étudiante au programme de doctorat en criminologie, chargée de cours et assistante de recherche au Groupe de recherche sur la question des femmes en criminologie dirigé par mesdames Marie-Andrée Bertrand et Louise Langelier Biron à l'Université de Montréal. L'auteure tient à remercier sincèrement madame Marie-Andrée Bertrand pour ses précieux conseils et suggestions. 


\section{PROBLÉMATIQUE ET MÉTHODE}

Notre recherche est exploratoire et s'intéresse au phénomène dans son ensemble. Sans nous limiter à un aspect précis du rapport des femmes à l'héroïne, nous avons d'abord entrepris de faire un bilan modeste, ce qui incitait à nous engager dans un mémoire documentaire.

Quelques interrogations toutefois nous guidaient. Premièrement, que prétend-on savoir des femmes consommatrices d'héroïne dans la littérature; deuxièmement, quelles sont les dynamiques sous-jacentes définies par les auteurs - de l'entrée et de la sortie du monde de l'héroïne dans le cas des femmes? Nos lectures et nos expériences (travail dans une prison pour femmes et dans un centre de traitement pour «toxicomanes») nous faisaient poser l'hypothèse d'une spécificité de cette dynamique dans le cas de ces femmes et nous nous préoccupions, par exemple, de savoir de quelle façon les auteurs en discutaient. Les auteurs avaient-ils toujours une approche «mixte», c'est-à-dire comprenant les femmes et les hommes? Pour des raisons que nous aborderons à la section 4, nous éviterons de parler ici d'héroïnomanie.

Le but de notre recherche étant de montrer de quelle façon on traite de la question de la consommation d'héroïne chez les femmes dans la littérature, deux possibilités s'ouvraient à nous pour retrouver les textes traitant de ce sujet: la méthode manuelle de recherche des documents et celle utilisant des bases de données bibliographiques.

De nombreux avantages (couverture internationale, mise à jour continuelle, variétés de documents, etc.) rendent nécessaire l'utilisation de la méthode de repérage de l'information faisant appel aux bases de données bibliographiques informatisées mais ne dispensent pas de compléter la collecte manuellement.

\section{DESCRIPTION DES BASES DE DONNÉES BIBLIOGRAPHIQUES UTILISÉES ${ }^{1}$}

Nous avons fait appel à neuf bases de données pour notre recherche. Elles proviennent d'un certain nombre de serveurs: DRUG de BRS (DRUG), FRANCIS de Questel, MEDLINE de la National Library of Medicine, NCJRS, PAIS INTERNATIONAL, PASCAL M de Questel, PSYCHINFO, REPERE d'INST-Informatique de SOCIOLOGICAL ABSTRACTS de Dialog Information Services.

1. Les renseignements factuels de cette section sur la description des bases de données proviennent des sources suivantes: Monique Berthiaume, Revue des documents écrits sur les professeurs des universités, miméo, 1984, 29 pages; Data Base Directory, printemps 1986; et Directory of Online Databases, janvier 1987. 
Dans cette étude, nous interrogeons des bases de données informatisées en employant la recherche booléenne et l'opérateur logique «et» donnant lieu à l'intersection des classes «femmes» et «héroüne».

Pour les fins de cet article, nous présenterons la description, l'énoncé de la recherche, et les résultats obtenus pour l'une de ces bases, DRUG, afin d'illustrer la stratégie de recherche utilisée. Ces étapes ont été reprises pour chacune de ces bases, on en voit l'effet au tableau 1.

La base de données bibliographiques (DRUG: Druginfo and Alcohol Use Abuse) est produite depuis 1968 aux États-Unis à l'Université du Minnesota, par le College of Pharmacy, Drug Information Services. Les sujets qu'elle couvre touchent les aspects éducationnel, sociologique, médical et psychologique de l'usage et de l'abus des drogues et de l'alcool.

En octobre 1985, DRUG contenait plus de 21000 références. On y indexe des documents des types suivants: articles de revues, documents et rapports gouvernementaux, monographies, livres, communications écrites soumises lors de conférences, documents audiovisuels, chapitres de livres, etc. Sa mise à jour se fait tous les trois mois. Aucun résumé n'accompagne les références. Les descripteurs se retrouvent dans un thésaurus.

L'énoncé de la stratégie de recherche effectuée peut se représenter par le schéma suivant ${ }^{2}$ :

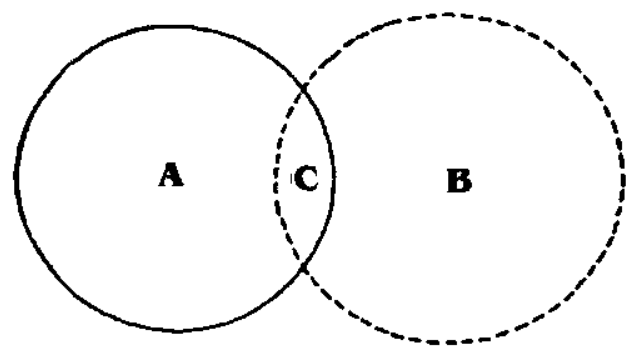

Les références pertinentes sont celles se trouvant à l'intersection de $\mathrm{A}$ et $\mathrm{B}$, c'est-à-dire dans la zone $\mathrm{C}$.

2. $\mathrm{A}=$ «héroin», «diacelytmorphine/de», «heroine dependence» ou «héroine»; B = «women», «woman», «femme», «female» ou «human female».

Les propositions des ensembles permettent seulement des comparaisons intra-bases et non inter-bases. 
TABLEAU 1

Résultats de la consultation des neuf bases de données bibliographiques

\begin{tabular}{lccc}
\hline Base consultée & $\begin{array}{l}\text { Nb de références } \\
\text { à l'héroïne }\end{array}$ & $\begin{array}{l}\text { Nb de références } \\
\text { à l'héroüne } \\
\text { et à la femme }\end{array}$ & $\begin{array}{l}\text { \% de références } \\
\text { à l'héroïne } \\
\text { et à la femme }\end{array}$ \\
\hline Drug & 664 & 46 & 6,9 \\
Francis & 33 & 1 & 3,3 \\
Medline & - & 71 & - \\
NCJRS & 633 & 65 & 12,6 \\
$\begin{array}{l}\text { Pais } \\
\text { international }\end{array}$ & 56 & 2 & 3,6 \\
Pascal M & 540 & 15 & 2,8 \\
Psycinfo & 1302 & 23 & 1,9 \\
Repere & 63 & 0 & 0 \\
$\begin{array}{l}\text { Sociological } \\
\text { abstracts }\end{array}$ & 194 & 34 & 17,5 \\
Total & 3485 & 257 & $7 \%$ \\
\hline
\end{tabular}

L'ensemble $\mathrm{A}$, représentant les références à l'héroöne contenait 664 documents, l'ensemble $\mathrm{B}$ à peu près $831^{3}$, tandis que l'intersection des ensembles $A$ et $B$ donne 46 références. Ainsi, on peut constater que les références portant à la fois sur l'héroïne et les femmes ne comptent que pour $6,9 \%$ du nombre total portant sur l'héroïne.

Plus de 250 références pertinentes ont été obtenues dans les neuf bases de données, ce qui ne représente toutefois que $7 \%$ de la production consacrée au sujet héroïne.

À l'analyse, nous avons dû constater que près de $50 \%$ des références se retrouvent dans plus d'une base de donnée: le repérage les amène en double ou même en triple, ce qui réduit le nombre d'oeuvres se portant sur la relation femmes et héroïne. Au terme du processus de triage, nous n'obtenons qu'un peu plus de 125 références distinctes. De

3. Dans la base DRUG, on trouve 67 références sous le descripteur "woman» et 831 références sous «women». Ces chiffres ne peuvent être additionnés car il faut tenir compte des dédoublements dont nous n'avons pas fait le compte. Les bases MEDLINE, NCJRS, PAIS INTERNATIONAL et SOCIOLOGICAL ABSTRACTS présentent des difficultés semblables. Aussi laisserons-nous l'ensemble B («women»...) en pointillé dans ce cas. 
l'avis des spécialistes en sciences de l'information et en bibliothéconomie, ce sont des effets techniques qui produisent ces doublets ou triplets et non l'importance d'une oeuvre, nécessairement. À titre d'illustration, disons qu'un auteur $\mathrm{X}$ écrit dans une revue $\mathrm{Y}$, une oeuvre $\mathrm{Z}$; si cette revue est inventoriée par plusieurs bases de données bibliographiques, on retrouvera l'oeuvre $\mathrm{Z}$ dans trois ou quatre bases, par exemple.

À la suite d'un premier déblayage puis d'un regard plus analytique, nous avons dégagé ce qui nous est apparu comme les aspects clés de la question, en procédant par induction.

Selon nous, ces écrits se regroupaient autour des thèmes suivants:

1) étendue de l'usage;

2) caractéristiques des femmes consommatrices d'héroïne et facteurs d'engagement dans la pharmacodépendance;

3) le traitement et

4) la mère consommatrice d'héroïne et ses enfants.

\section{UN BILAN DES CONNAISSANCES SUR LES FEMMES CONSOMMATRICES D'HÉROÏNE}

Les rubriques déjà annoncées seront utilisées comme sous-titres dans cette section.

\section{1 ÉTENDUE DE L'USAGE}

Par l'étendue de l'usage, nous nous référons à un des aspects de l'épidémiologie, à la prévalence du phénomène, c'est-à-dire combien de personnes, dans ce cas-ci combien de femmes, consomment de l'héroïne. Ce fait n'a pas toujours été un objet de préoccupation sociale ni défini comme illégal quand on regarde dans le temps ou dans l'espace. Comme le fait constater Thomas Szasz:

C'est une réalité historique qu'avant 1914 , il n'existait pas de «problème de drogue» aux États-Unis et nous ne lui avions pas donné un nom. Aujourd'hui, il existe un problème monumental de la drogue aux États-Unis (...). ce que je veux dire, c'est que nos experts de la toxicomanie, nos législateurs, nos psychiatres et autres gardiens professionnels de la morale médicale ont fabriqué des couveuses à poussins et ils continuent (grâce en partie à certains abus caractéristiques et tactiques du langage) à fabriquer et entretenir le "problème de la drogue» qu'ils prétendent ostensiblement résoudre (...).

(Szasz, Thomas, 1976: pp. 33-34). 
Dans la perspective qui nous intéresse, qui est celle de l'étendue de l'usage, la mesure du phénomène est elle-même une construction sociale, pour reprendre un terme cher à Becker (1963). Non seulement on fabrique la mesure mais elle est fortement conditionnée par le climat social. Szasz (1976) va même jusqu'à comparer les programmes antidrogues aux chasses aux sorcières à une autre époque. De tout temps, c'est la recherche de boucs émissaires.

Il n'est cependant pas inutile de comparer les relevés officiels de l'étendue de l'usage de l'héroïne. Nous jetterons un coup d'oeil sur les chiffres en France et aux États-Unis et nous nous attarderons un peu plus à ceux du Canada.

Dans les statistiques officielles de la France, on estime que le nombre de «consommateurs-trices d'héroïne» connu(e)s pour ce pays est de 80000 à 120000 (communication donnée à Montréal par Marc Valleur, médecin-adjoint au Centre médical Marmottan, à Paris, juin 1987).

Aux États-Unis, pour la seule ville de New York, les chiffres officiels portent à 250000 le nombre des «héroïnomanes» connus (statistiques rapportées par le Beth Israel Medical Center, 1986). La majorité d'entre eux sont encore principalement des hommes mais la proportion des femmes devient de plus en plus importante. Elles représentent environ 20 à $30 \%$ de la population de consommateurs d'héroïne (Ellinwood, Smith et Vaillant, 1966; Hambenger, 1969; Mandell, Goldsmith et Drover, 1973; Bureau des drogues dangereuses, 1985; New York State of Substance Abuse Services, 1986).

Au Canada, le Bureau des drogues dangereuses (1985) indique que le nombre d'«usagers(ères) d'héroïne» connu(e)s s'élève à 2207 pour les hommes et 1068 pour les femmes, c'est-à-dire une femme pour deux hommes. Si nous analysons ces chiffres pour les quatre provinces (Québec, Ontario, Alberta et Colombie-Britannique) qui comptent le plus grand nombre d'usagers d'héroïne, le rapport femmes/hommes varie légèrement entre 1 contre 2 et 1 contre 3 , ou un peu moins, ce qui $s$ 'approche du rapport femmes/hommes aux États-Unis.

Selon les auteurs consultés et au vu des statistiques, nous pouvons dire que la consommation de drogues varie selon le sexe, et se présente différemment selon qu'il s'agit de drogues licites ou illicites. Les données officielles font état d'une différence dans la consommation selon le sexe: les femmes abuseraient plus de substances licites mais la consommation de substances illicites serait davantage le fait des hommes. 
Ferrence et Whitehead (1980) recourent à quatre modèles et classent les auteurs selon leur grille explicative pour rendre compte de ces différences. Le premier qu'ils appellent le «modèle de la déviance» explique la répartition inégale des sexes pour ce qui touche la consommation de drogues illégales du fait que les femmes seraient moins «déviantes» que les hommes, ce qui concorde avec ce que disent d'autres auteurs (Chein, Gérard, Lee et Rosenfeld, 1964; Sutker, Archer et Allain, 1980). Selon Cuskey, Prenkumar et Sigel (1974), on comptait plus de femmes consommatrices de morphine et d'héroïne que d'hommes avant la proclamation du Harrison Act en 1914 aux ÉtatsUnis. La «criminalisation» de simple possession aurait conduit les femmes vers un réseau de consommation plus conformiste.

Un autre modèle d'explication peut nous être utile pour comprendre la place des femmes dans la consommation de drogues illicites: il s'agit du «modèle de diffusion de l'innovation». Selon ce modèle, les premiers à adopter un comportement auraient tendance à être de statut social élevé, d'un haut niveau d'éducation, à être plus «exposés» aux médias, plus en contact avec des agents de changement social et avoir une plus grande participation dans la société (Bell et Champion, 1976). C'est donc dire, toujours selon Ferrence et Whitehead, que les hommes s'adonnent à la consommation de drogues plutôt que les femmes. Ce modèle a été utilisé pour expliquer, par exemple, les récents développements du tabagisme. Des auteurs avancent que le tabagisme a diminué chez les hommes mais a augmenté chez les femmes (Bakal, Rootman et Campbell, 1976; Smart, Fejer, Smith et White, 1975; Smart, Goodstadt et Sone, 1977; Blackford, 1977).

Un troisième modèle peut nous aider à situer la consommation d'héroïne chez les femmes, celui du «réseau social». Ferrence et Whitehead estiment que ce sont les hommes qui initient les femmes à la consommation. Nous assimilerons à cette catégorie explicative plusieurs auteurs qui estiment que l'initiation des femmes à l'usage des drogues - quelle que soit la drogue - se fait par un homme. Il semblerait que le fait d'être mariée à un homme toxicomane entraînerait la toxicomanie chez la femme mais l'inverse n'est pas nécessairement vrai (Suffet et Brotman, 1976). Howard et Borges (1970) dans leur recherche estiment que $68 \%$ des hommes s'injectent eux-mêmes leurs drogues tandis que seulement $29 \%$ des femmes le font elles-mêmes. Cette recherche laisse entendre que ce sont les hommes qui «injectent» les drogues aux femmes. 
Revenant à Ferrence et Whitehead, leur quatrième modèle serait le «modèle socio-économique» qui avance l'hypothèse qu'il y aurait un lien entre le statut socio-économique élevé, le type et le niveau d'usage de drogues. Cette hypothèse pourrait permettre d'avancer que, puisque les femmes (en général) ont un statut socio-économique inférieur à celui des hommes, elles auraient une consommation de drogues illicites également inférieure. De plus, les auteurs comparent les femmes entre elles. Ils estiment que si le statut socio-économique joue un rôle sur la consommation de drogues, les femmes ayant un revenu, par exemple, feront un usage plus grand de drogues que celles qui n'ont pas de revenu. En fait, plus les femmes auront un revenu élevé, plus leur consommation de drogue sera grande. Toujours selon les auteurs, les différences de consommation entre les sexes varieront proportionnellement avec le prix des drogues consommées. Les différences de sexe se manifesteront davantage dans l'usage de drogues relativement dispendieuses, comme l'héroïne, que dans celui de drogues relativement «bon marché», comme la marijuana.

Ces quatre modèles (de déviance, de diffusion de l'innovation, du réseau social et socio-économique) peuvent donc nous éclairer sur l'étendue de l'usage d'héroïne chez les femmes.

\subsection{CARACTÉRISTIQUES DES FEMMES CONSOMMATRICES D'HÉROÏNE ET FACTEURS D'ENGAGEMENT DANS LA PHARMACODÉPENDANCE}

Les femmes consommatrices d'héroïne sont décrites comme posant plus de problèmes au niveau de la dynamique et de l'intervention.

Dans leur recherche, Baldinger, Capel, Goldsmith et Stewart (1973) arrivent aux conclusions suivantes concernant les femmes consommatrices d'héroïne, comparées d'une part à des non-consommatrices, et d'autre part, à des consommatrices de marijuana:

1. il y a une distance importante entre les attitudes prises dans la "vraie vie» et le discours tenu par les consommatrices d'héroïne au cours des entrevues menées dans le cadre de cette recherche;

2. elles entretiennent des stéréotypes sexuels quant au rôle féminin contrairement à ce que l'on peut penser;

3. les consommatrices d'hérö̈ne privilégient des valeurs associées aux sentiments interpersonnels et intrapersonnels, tandis que les hommes consommateurs d'héroine mettent l'accent sur des valeurs reliées au succès et à la compétence; 
4. malgré leur style de vie déviant, elles possèdent et expriment des valeurs plutôt traditionnelles et conventionnelles.

D'autres auteurs ajoutent que les consommatrices d'héroïne sont plus préoccupées que leurs homologues mâles par des valeurs telles la propreté et le respect de soi; selon ces auteurs, les consommatrices veulent ainsi annuler un sentiment de malpropreté et d'être sans valeur, sentiment associé à leurs expériences de vie (Miller, Sensenig, Stocker et Campbell, 1973).

D'autres rapportent que les femmes narcomanes en traitement ont besoin de beaucoup d'attention (Ellinwood, Smith et Vaillant, 1966).

Selon Colton (1977), les femmes consommatrices d'héroïne ont une faible capacité d'affirmation de soi («weak-assertiveness»), qui résulterait probablement de leur intégration dans une sous-culture déviante mâle. Cette intégration leur occasionne encore plus de stigmatisation (in Cuskey et Wathey, 1982). Dans ce même ordre d'idée, Arnon, Kleinman et Kissin (1974) soutiennent qu'étant donné que les hommes vivent une plus grande pression des pairs (pour consommer de l'héroïne) que les femmes, cela pourrait expliquer que l'utilisation de drogues constitue chez eux une plus faible déviance sociale que chez les femmes.

Nous regrouperons quelque soixante oeuvres supplémentaires autour des thèmes suivants: 1) la psychopathie, les traits névrotiques et la dépression; 2) l'image et l'estime de soi et 3) le support social.

Dans ces oeuvres, les femmes sont perçues comme étant plus malades que les hommes ou présentant des pathologies différentes.

\section{1) «Psychopathie» traits névrotiques et dépression}

Sutker, Archer et Allain (1980) ont mesuré les dimensions de psychopathie au «Minnesota Multiplasic Personality Inventory» (MMPI) sur 428 «toxicomanes» (342 hommes et 86 femmes). Selon leur recherche, la majorité des sujets est caractérisée par une «psychopathie» décrite comme une personnalité anti-sociale, «passive» et dépendante avec des traits anti-sociaux et caractérisée par des variantes d'une symptomatologie psychotique. Les résultats ne démontrent, entre autres, aucune différence significative au niveau des dimensions cliniques ou types de psychopathie, selon le sexe. Pour une analyse plus complète, voir Sutker, Archer et Allain (1980), «Psychopathologie of Drug Abusers : Sex and Ethnic Considerations». 
Pour Ellinwood, Smith et Vaillant (1966), les femmes consommatrices d'héroïne sont plus névrotiques et psychotiques tandis que les hommes ont plutôt des troubles de personnalité et sont sociopathiques.

Les données de Cuskey et Walthey (1982) dans Female Addiction, n'appuient pas l'hypothèse que la toxicomanie des femmes serait plus pathologique mais que ces dernières seraient plus disposées à la dépression et à une faible estime de soi. Un nombre considérable de rapports cliniques font ressortir la dépression comme fortement associée à l'assuétude aux opiacés en se basant le plus souvent sur le MMPI.

Une faiblesse commune à ces oeuvres consiste en ce que les auteurs ne disent pas si ces traits et facteurs précédaient la pharmacodépendance, l'accompagnaient ou y succédaient. Il est difficile de dissocier la «personnalité» des effets de traitement, par exemple.

\section{2) Image et estime de soi}

Selon Leventhal (1983) chez les toxicomanes des deux sexes, on observe une distorsion dans l'image corporelle mais cette distorsion est différente selon le sexe. Mais encore là, les oeuvres ne nous permettent pas d'établir si cela s'est produit avant, pendant ou après la toxicomanie. Il est à noter que les distorsions rapportées par les femmes avaient tendance à les inférioriser, tandis que celles rapportées par les hommes avaient tendance à les mettre à leur avantage.

Gossop (1976) démontre que les femmes toxicomanes ont une plus faible estime de soi que leurs homologues mâles et que les femmes non-consommatrices. Les toxicomanes des deux sexes auront une plus faible estime de soi qu'une population non-toxicomane.

\section{3) Support social}

Il existe de nombreux écrits et quelques recensions sur le «support sóciàl» et son absence comme facteur de dépendance à la drogue (Cassell, 1976; Cobb, 1976; Pinneau, 1976; Gore, 1978; Caplan, 1979; House, 1980; Kahn et Antonucci, 1980). Belinda Tucker (1982) est l'auteure d'une recension de plusieurs études s'intéressant à la question $\mathrm{du}$ «support social» en rapport avec la toxicomanie.

Dans cette recension, on voit qu'en absence de support, les femmes plus que les hommes avaient recours à des stratégies non-sociales et dysfonctionnelles pour faire face à la colère et à la dépression. Les hommes n'avaient jamais recours à ces stratégies en l'absence d'alternatives sociales. 
Les conclusions auxquelles arrivent les auteurs qui s'intéressent aux caractéristiques et facteurs nous semblent émaner d'une perspective surtout psychologique.

\subsection{LE TRAITEMENT}

Le traitement est un thème privilégié dans les écrits. Trois remarques s'imposent à l'égard de ces productions: 1) de façon générale, les auteurs s'intéressent presqu'exclusivement à l'abstinence et à la cure d'entretien à la méthadone comme formes de traitement. Pour ce qui est de la méthadone, on peut l'expliquer par l'essor qu'a connu cette substance dans les années 70 (date qui concorde avec la parution d'une masse d'écrits); 2) pour ce qui touche les femmes plus particulièrement, les auteurs se préoccupent surtout des femmes enceintes et 3) ils s'intéressent surtout aux effets de la drogue sur le foetus. Nous ne nous attarderons pas aux oeuvres qui prennent en compte la grossesse et le foetus, pour mettre davantage le focus sur la femme.

\section{Le processus d'engagement dans la "cure»}

L'analyse des raisons (de consommer et d'arrêter) est importante car elle permet de comprendre les forces qui amènent la consommation d'un côté et de l'autre, ce qui en déclenche l'arrêt.

Les raisons (d'entrer ou de sortir de la consommation d'héroïne) peuvent varier selon le sexe. Une étude de Brown, Gauvey, Meyers et Stark (1971) montre que les hommes citent la curiosité comme raison majeure de leur premier usage d'héroïne, tandis que pour ce qui est des femmes, les facteurs suivants dominent: 1) l'influence des amis (ou amies, non précisé); 2) la curiosité; 3 ) le soulagement des perturbations personnelles. En ce qui concerne le premier arrêt de la consommation d'héroïne, les hommes ont mentionné comme première raison : changer leur façon de vivre et de fonctionner et comme deuxième, des problèmes de santé et d'argent. La moitié des femmes invoquaient comme première raison les problèmes de santé reliés à la consommation de l'héroïne. Ces raisons (d'entrée ou de sortie) peuvent avoir un impact sur le traitement.

Certaines oeuvres s'interrogent sur la place des femmes dans les communautés thérapeutiques mais la cure d'entretien à la méthadone occupe une place de choix dans la littérature. 


\section{La méthadone : un peu d'histoire}

En 1972, le président des États-Unis proclamait que l'abus de drogues était le problème de l'heure et le gouvernement de l'époque a fait proliférer nombre de traitements différents: communautés thérapeutiques, centres de désintoxication, désintoxication à la méthadone et entretien à la méthadone (Nelken, 1973).

La méthadone a été développée pendant la Seconde Guerre mondiale par un Allemand, G. Farben, comme un anesthésiant synthétique. Cette substance a été prescrite pour faciliter la désintoxication des héroïnomanes dès 1948. Mais ce n'est que depuis 1963 qu'on l'utilise comme cure d'entretien prolongé de l'«héroünomanie».

Vers la fin des années 60 , les «pionniers» dans le domaine du programme d'entretien à la méthadone, les docteurs Vincent Dole et Marie Nyswander, commencèrent à faire rapport de leurs résultats de recherche. Ces résultats supportent l'idéologie de base qui a fait proliférer ces programmes tels qu'on les connaît aujourd'hui. En résumé, disons que ces «pionniers» postulaient que cette substance pouvait pallier le désordre métabolique créé par la dépendance à l'héroïne et qu'une dose suffisante de méthadone pouvait empêcher le désir ou «craving» pour les opiacés; l'individu serait ainsi immunisé contre les effets euphoriques de l'héroine, n'entretiendrait plus de lien avec le monde de l'héroïne et deviendrait un membre «productif» de la société.

Bien que cette utilisation de la méthadone n'en était qu'au stade expérimental, les médias la qualifiaient de «drogue de Cendrillon»et on avançait que le problème de drogue serait ainsi réglé.

Le programme d'entretien à la méthadone offrait plusieurs avantages. Dole et Nyswander (1965) soulignent que : 1) la drogue peut se prendre par voie orale; 2 ) elle a un effet prolongé, de 24 à 36 heures; 3) pas d'effets secondaires sérieux à des doses d'entretien; 4) à des doses suffisantes, la méthadone bloque l'effet de l'héroïne; 5) administrée de façon thérapeutique, la méthadone ne produit pas d'euphorie. Un autre facteur important selon Nelken (1973): la méthadone était peu coûteuse.

Comme on peut le constater, avec la venue de la méthadone, la dépendance à l'héroïne devenait une maladie et un problème médical et l'«héroïnomane» devint un patient.

Les programmes d'entretien à la méthadone se sont multipliés. Après un début officiel en 1963 avec deux patients de Dole et 
Nyswander en mars 1965, ils connurent une extension au Beth Israel Medical Center avec six patients; dès 1968, on comptait 1139 patients dans les programmes; en décembre 1971, on estimait le nombre des patients aux États-Unis à 25000 (Brecher, 1972). En 1978, les chiffres grimpaient à 78000 (National Institute on Drug Abuse, 1978). En 1980, on en comptait 67851 . Et selon le rapport annuel du Beth Israel Medical center (1986), on compterait en 1986 plus de 80000 patients traités à la méthadone.

\section{La méthadone et les femmes}

Des auteurs se sont penchés sur cette forme de traitement en regard des femmes (Bahna et Gordon, 1978; Kail et Lukoff, 1984a, 1984b). Nous nous attarderons ici à une contribution de Marsha Rosenbaum (1982) qui s'intéresse au processus qui mène les femmes vers cette forme de traitement.

Pour cette auteure, les contraintes liées au style de vie qu'il faut mener pour se procurer l'héroïne deviennent intolérables (vie centrée sur la drogue, coût de la drogue, qualité de la drogue problématique, illégalité) et poussent ces femmes à aller vers la méthadone. Elles veulent sortir de cette vie, régler leurs problèmes de santé (problèmes reliés aux multiples injections, surdosage, grossesse) et protéger les enfants de cette réalité. En fait, elles ont peur que ces derniers ne prennent conscience de leur dépendance. Donc, c'est le style de vie qui devient intolérable et non l'usage d'héröne comme tel.

Cette auteure et plusieurs autres rapportent les propos des consommateurs à l'effet que la méthadone n'est utilisée qu'en dernier recours, c'est la «dope welfare», c'est se soumettre au contrôle de quelqu'un, c'est un échec. Selon Rosenbaum, le fait que la méthadone représente un échec ou un appel à l'aide peut nous expliquer pourquoi en 1979 à San Francisco plus de la moitié de la clientèle était des femmes (sic). Enfin, toujours selon cette auteure, la méthadone permet à ces femmes de rester avec leurs enfants contrairement aux autres formes de traitement.

\section{Les modèles dominants de traitement : des modèles mâles}

Beth Glover Reed (1985) estime que les femmes sont considérées comme un groupe minoritaire dans les programmes d'aide. Les services ne sont pas conçus de façon à répondre aux besoins des femmes et ne paraissent pas utiles aux femmes; ces programmes peuvent ne pas reconnaître les problèmes propres aux femmes. 
Selon Beth Glover Reed, la littérature concernant les femmes toxicomanes occupe très peu de place avant les années 70 . Elles étaient considérées (et le sont encore par certains auteurs) comme plus «malades», plus difficiles à traiter, moins motivées au traitement que les hommes, sexuellement plus déviantes ou autrement déviantes que les hommes, etc. Ces stéréotypes, toujours selon cette auteure, masquent le manque d'intérêt et de connaissances des planificateurs et responsables de programmes de traitement en ce qui touche la question des femmes.

Dans ce sens, des auteurs (Kail et Lukoff, 1984b; Reed, 1985, etc.) s'entendent pour dire que les programmes d'aide doivent être uniquement centrés sur une clientèle féminine plutôt qu'une clientèle prétendument mixte, mais en fait masculine. Les raisons suivantes alimentent leur prise de position: les programmes qui s'adressent aux femmes sont calqués sur le modèle masculin, le sexisme dans le traitement, la nécessité d'une approche différente, les problèmes et besoins propres aux femmes, la sous-représentation des femmes dans ces programmes (rations de 2:1 jusqu'à 10:1), etc.

La réalité des femmes, selon ces auteurs, appelle une intervention différentielle.

\subsection{LA MÈRE CONSOMMATRICE D'HÉROÏNE ET SES ENFANTS}

Dans cette partie, nous survolerons les principales études se préoccupant: 1) de la relation mère-enfant; 2) des habiletés parentales et 3) de la négligence et de l'abus des enfants.

Selon une recension de Beschner et Thompson (1981), 67 à 73\% des femmes en traitement pour leur toxicomanie ont des enfants. Colton (1980) avance que $50 \%$ des mères consommatrices d'héroïne ont leurs enfants ou certains d'entre eux avec elles au moment de l'entrée en traitement, situation moins fréquente chez les hommes toxicomanes et/ou consommateurs d'héroöne.

\section{1) Relation mère-enfant}

À propos des facteurs génétiques liés à la consommation de drogues chez l'enfant, les auteurs soulignent trois choses : a) l'enfant d'une mère héroïnomane manifestera des «désordres» de comportement; b) ces «désordres», à l'origine génétiques, affecteront la relation mèreenfant et c) il faut tenir compte des composantes psychologiques et sociales de cette relation pour avoir un portrait plus complet de la situation (Deren, S., 1986). 
Les difficultés suivantes rendent, entre autres, la relation mèreenfant encore plus ardue: les bébés manifestent certains «désordres», les mères sont souvent sans emploi, ont une faible estime de soi; elles sont anxieuses et dépressives selon une étude de NIDA (1980).

\section{2) Habiletés parentales}

Selon une étude de Colton (1980) les mères pharmaco-dépendantes se sentent plus souvent inadéquates dans leur rôle de mère, même s'il ne ressort pas de différence majeure dans leur relation avec l'enfant quand on les compare avec des mères non-consommatrices.

Pour Tucker (1979), ces mères qui ont des difficultés parentales avaient un réseau de support social inadéquat, rapportaient avoir moins d'ami(e)s et se sentaient seules.

Pour Deren (1986), les difficultés au niveau des habiletés parentales s'expliqueraient non seulement par le fait de la consommation de drogues, mais bien plus par les conséquences psycho-sociales de celleci et des caractéristiques personnelles antérieures entraînant une perception négative de soi.

\section{3) La négligence et l'abus de l'enfant}

La question du lien entre l'abus des enfants et l'abus de drogue ou d'alcool a été soulevée fréquemment dans la littérature, mais comme le fait ressortir Deren (1986), on l'a associée à une foule de caractéristiques qui amènent un parent à être «à risque»: pas d'emploi, pauvreté, manque de modèle d'apprentissage parental, etc.

\subsection{LA QUESTION DU SIDA ET CELLE DE LA PROSTITUTION}

Un mot à propos du sida: selon les auteurs recensés, le facteur déterminant de la transmission du sida pour les femmes $(52 \%)$ est l'usage intraveineux de drogues. Pour Guinan et Hardy (1987), il y a une hausse de l'incidence du sida chez les femmes en général: les chiffres passent de $12 \%$ de femmes atteintes du sida en 1981 à $26 \%$ en 1986.

Il est apparu particulièrement urgent et important de s'intéresser à la question du sida chez les consommatrices d'héroöne, parce que : 1) ces femmes peuvent transmettre l'infection HIV au nouveau-né et 2) ces femmes qui sont souvent associées, dit-on, à la prostitution, peuvent transmettre le virus à leurs clients et peuvent le recevoir. 
Ce qui nous amène à replacer cette question du sida dans le contexte plus large de la prostitution et de la déviance reliée à la dépendance à l'héroöne. Plusieurs auteurs se sont penchés sur cette question (Datesman, 1980; Miller, 1980; Blorent, 1982; Inciardi, Pottieger et Faupel, 1982; Covington, 1985; Sanchez et Johnson, 1986). Inciardi (1980) avance que seulement $15 \%$ des femmes consommatrices d'héroïne étaient impliquées dans la prostitution, ce qui est corroboré par Pottieger et Faupel (1982). Il ne faut toutefois pas nier ce type de comportement sauf que l'intérêt pour la question est davantage lié à une réaction sociale donnée.

\section{CONCLUSION ET INTERPRÉTATION CRITIQUE DES RÉSULTATS}

Plusieurs constatations majeures s'imposent concernant les productions analysées. La quantité de la littérature sur la question des femmes représente un faible pourcentage de la littérature sur l'héroïne (7\%). La qualité de certaines de ces recherches peut être mise en doute et certaines critiques peuvent être classées sous trois catégories: 1) les problèmes de méthode et d'échantillon (fiabilité, fidélité, populations étudiées...); 2) les problèmes épistémologiques: problèmes de valeur, problème de qualification morale d'un phénomène et donc absence de position critique des auteurs par rapport à la relativité de la norme, problème de «modèle» et de paramètre (les analyses faites sur des populations masculines engloberaient, selon les auteurs, les femmes). Dans ce même ordre d'idée, il y a confusion dans les termes: usage devient abus et consommation devient dépendance et 3 ) les problèmes issus du fait que les études sont souvent monodisciplinaires, alors que le phénomène étudié appelle une perspective interdisciplinaire.

Une réserve fondamentale mérite d'être discutée concernant la qualité : la population qui constitue très souvent la référence de base est composée d'hommes. Ce qui est identifié comme la norme ou la «normalité» (de la toxicomanie, de son incidence, de ses effets, de ses remèdes ...) est le fait du sexe masculin; très souvent, les femmes seront considérées comme en marge... de cette norme.

... le sexe féminin est conçu comme celui qui fait problème, qui n'est pas comme tout le monde.

(Dhavernas, M.J., 1978: 78).

En fait, elles sont marginales dans leur marginalité (Dhavernas, M.J., 1978). Schur (1984) va même jusqu'à dire qu'elles sont margi- 
nales du fait qu'elles sont femmes. En criminologie, notamment, le problème a été posé avec les études comparatives «délinquant» versus «non-délinquant» qui posent aussi le problème de la norme. Nous n'avons qu'à penser, entre autres, aux travaux de Becker, 1963; Matza, 1964 et de Taylor, Walton et Young, 1973, etc.

Ce qui paraît constituer la faiblesse fondamentale de certaines de ces études, ce sont des problèmes d'ordres méthodologique, idéologique et épistémologique. Les modèles sont sexuellement déterminés, ce qui vicie les paramètres.

De plus, ces études privilégient souvent une approche très «psychologisante»: les problèmes sont propres à l'individu. D'autres apports comme ceux de la sociologie, de la politique, de l'économique, du juridique, de l'anthropologique, etc. restent inexploités. À notre avis, il faudrait adopter une vision pluraliste, interdisciplinaire.

Autre problème: les concepts utilisés pour définir la dépendance répondent à des critères moraux ou psycho-sociaux, plutôt que pharmacologiques (posologie) ou toxicologiques (toxicité). Les concepts ne sont pas univoques pour tous les auteurs, ce qui limite sérieusement les comparaisons entre les études.

Une autre critique: les auteurs ne font pas ressortir la relativité des normes. Ici, c'est le caractère illégal de l'héroüne qui détermine les constatations négatives, beaucoup plus que des considérations d'ordre pharmacologique comme on le prétend. Dans notre société, on n'admet pas qu'un individu puisse avoir une consommation «contrôlée» d'héroïne, au même titre qu'un autre prendra seulement quelques verres d'alcool. Notons que certains auteurs abordent cette question: Zinberg, Jacobson et Harding, 1975; Zinberg et Jacobson, 1976; Harding, Zinberg, Stelmack et Barry, 1980. De plus, tout le «folklore» qui entoure la question de l'héroïne nourrit la conception erronée qui veut que tout usage soit un abus, que tout usage entraîne le passage dans un monde d'interdits.

Il faut donc aborder la question de fond: qu'est-ce qui fait qu'une drogue est «légale» et une autre «illégale»? Cette distinction ne se fonde pas sur des critères d'ordre pharmacologique.

En fait, l'arbitraire apparent qui sépare la drogue du médicament, semble souvent correspondre pour une certaine part à l'idéologie en cours.

Là encore, les mécanismes de définition sociale obéissent aux impératifs qui régissent la production des normes (...). La drogue 
«mauvaise figure» que notre société ne sait pas intégrer, reste un reflet du temps (...). En fait, ses seuils de tolérance sont nécessairement variables selon les époques et les sociétés.

(Mandon, D., 1984: 167).

Nous estimons qu'il faut souligner le caractère normatif du monde de la toxicomanie, ce que les auteurs ne font pas suffisamment.

L'approche anthropologique peut nous éclairer ici. Il faut bien dire que les études répertoriées dans ce mémoire avaient comme toile de fond l'Amérique du Nord principalement; ce contexte particulier ne peut rendre compte de la réalité transculturelle et plus précisément de la consommation de toxiques par les femmes, dans toutes les sociétés:

(...) l'incidence de l'alcoolisme et de la toxicomanie est plus grande chez les hommes que chez les femmes. Cette différence fréquemment observée dans les sociétés occidentales a conduit à une croyance que dans toute société, les hommes consomment pius d'alcool et utilisent plus de produits toxicomanogènes que les femmes. Les évidences transculturelles indiquent que tel n'est pas le cas (...).

(Cardinal, N., $1984: 201-202$ ).

La même auteure ajoute que lorsque la consommation connue d'alcool et de toxiques par les femmes est inférieure à celle des hommes, on peut expliquer cette différence par des facteurs extrinsèques:

La distribution différentielle d'alcool par sexe s'explique donc en bonne partie par le contrôle social exercé et par le contexte favorisant une consommation masculine.

(Op. cit., 1984: 202).

En plus des différences culturelles, il faut tenir compte des variations temporelles. Comme le constate Thomas Szasz, dans Les rituels de la drogue (1976), l'usage d'une drogue peut à un moment donné être approuvé, encouragé, et à un autre, banni.

En fait, la seule chose dont on peut être assuré c'est que les toxicomanes et ici les consommateurs d'héroïne n'ont en commun qu'un usage dit «inapproprié» de drogue. C'est à ce titre que l'on parle de la loi de Keller (1972) qui avance que les individus qui ont des difficultés liées à l'alcool notamment, n'ont rien en commun sauf une consommation «inappropriée» d'alcool. Selon cette règle, les femmes «hérönomanes» n'ont en commun que cette caractéristique et sont différentes sur d'autres traits, par ailleurs, majeurs.

D'autre part, les termes héroïnomane, toxicomane, narcomane, doivent être remis en question. Il convient de présenter le problème 
qu'ils posent. Sontag (1979) estime qu'il est dangereux d'utiliser des attributs qui s'avèrent réducteurs et qui réfèrent à une identité personnelle. Les auteurs du DSM III, Manuel diagnostique et statistique des troubles mentaux (1983) croient qu'une classification des troubles mentaux ne peut servir à classer des personnes.

À l'instar de Sontag (1979), il faut reconnaître le caractère réducteur, stigmatisant et indûment généralisateur de ces étiquettes qui tentent d'englober toute la personne sous la bannière du comportement.

À la lumière de ces diverses critiques concernant les populations étudiées, les méthodes utilisées dans plusieurs études, les présupposés théoriques de certains autres, les concepts et la «norme» de référence, il convient de s'interroger sur le type de recherche et les modèles à privilégier. Nous en sommes arrivées à deux suggestions.

La première envisage l'idée de construire un modèle de recherche qui privilégierait l'étude des femmes consommatrices d'héroïne en tant que groupe, c'est-à-dire qui s'intéresse aux femmes les unes par rapport aux autres plutôt qu'en regard des hommes. Ainsi, on étudierait le cas des femmes consommatrices d'héroïne selon leur classe sociale, leur occupation, leur âge, leur niveau éducationnel, leurs antécédents personnels, familiaux et sociaux. Cette proposition devrait par ailleurs favoriser l'émergence de pistes ne se limitant pas aux apports de la psychologie, mais englobant les perspectives sociologique, anthropologique, etc.

La deuxième suggestion a pour objectif de construire une grille d'analyse plus générale de la marginalité, de la déviance et de la criminalité réprimée chez les femmes selon une perspective féministe. Cette grille pourrait offrir des pistes intéressantes en vue de la compréhension d'un type de conduite marginalisé, tel la consommation d'héroïne chez les femmes. On peut se demander par exemple, comment est représentée la déviance des femmes? Comment est-elle perçue par les agents de contrôle social? Quelles sont les conséquences de cette construction sociale? Des tentatives de réponses à ces questions nous apporteraient des éléments essentiels nécessaires si l'on veut saisir un phénomène spécifique de la consommation d'héroüne et son traitement. Une analyse féministe pourrait nous offrir un éclairage nouveau et novateur.

Il faut noter que ces deux suggestions ne sont pas mutuellement exclusives: l'une n'empêche pas l'autre, les deux pouvant être complémentaires. Nous commenterons plus en détail notre deuxième suggestion. 
Notre propre démarche intellectuelle et les questionnements suscités et alimentés par le GRFC (Groupe de recherche sur la question des femmes en criminologie) nous amènent à croire que c'est au croisement des théories de la réaction sociale en criminologie et du féminisme radical matérialiste, que l'analyse des problèmes sociaux qui confrontent les femmes nous semble devoir être la plus fructueuse.

Parmi les courants de pensée en criminologie, la théorie de la réaction sociale se démarque des théories étiologique, fonctionnaliste, et du conflit, en ce sens qu'elle renverse l'étude de la trilogie: individu déviance - régulation, pour mettre l'accent sur: régulation - déviance individu.

Pour les théoriciens de la réaction sociale (Robert, 1973; Black, 1976; Hulsman, 1977), l'ordre social est un ordre négocié, c'est une construction sociale. Il y a distribution inégale des biens mais surtout des pouvoirs de définition, du «labeling» par exemple. La régulation de la déviance se fait par des mécanismes de sélection, des pouvoirs discrétionnaires. Ces niveaux de régulation peuvent s'articuler ainsi: application discriminatoire de la loi, attribution des biens négatifs, «labeling» et processus de renvoi. C'est une entreprise morale. Ce sont des modes de régulation primaire et secondaire qui entretiennent ce processus. Le but de cette entreprise morale est de différencier, de mettre en catégorie. La théorie de la réaction sociale s'engage à infirmer la thèse de l'égalité devant la loi.

Cette description très sommaire de cette théorie laisse entrevoir les possibilités d'analyse quand il s'agit de femmes. D'autre part, le féminisme radical matérialiste présente des connivences avec la théorie de la réaction sociale, et apporte directement un éclairage sur la marginalité, la déviance et la criminalité des femmes. Selon DescarriesBélanger et Roy (1988), le féminisme radical matérialiste est une sousbranche du féminisme radical qui se divise en trois courants : matérialiste, de la spécifícité et des biens.

Le féminisme radical matérialiste, selon ces auteures, s'engage à faire ressortir «les mécanismes producteurs et reproducteurs de l'oppression" (p. 11). Les auteures associent à ce courant certaines oeuvres des auteures suivantes: d'Eaubonne, F., 1974; Dworkin, A., 1974; Brownmiller, S., 1975; Guillaumin, C., 1978; Barry, K., 1979; Griffith, S., 1984; French, M., 1985; Mathieu, N.C., 1985. Toujours selon Descarries-Bélanger et Roy, ce sont des oeuvres et non des auteures qui représentent l'unité d'observation, étant donné qu'un auteur peut traverser les courants de pensée féministes à divers 
moments de l'histoire. Pour le féminisme radical matérialiste, toujours selon Descarries-Bélanger et Roy (1988), l'oppression crée le sexe, ce qui nous renvoie à la théorie de la réaction sociale en criminologie. L'objet d'analyse en question pour ce courant, toujours selon les auteures, s'avère les phénomènes d'appropriation qui s'expriment par le contrôle social à l'endroit des femmes. L'apport de ce courant en criminologie, traitant de la réaction sociale et du féminisme radical matérialiste dans ce sens, peut nous éclairer dans cette deuxième piste, qui s'avère selon nous la plus prometteuse.

Ces pistes devraient permettre de mieux rendre compte de la réalité sociale de toutes les femmes - marginales, déviantes, consommatrices d'héroïne --, puisqu'elles passeraient par la représentation sociale qu'on s'en fait. Tel ne semble pas être le cas des productions repérées et analysées ici. Nous en sommes arrivés à la conclusion que la consommation d'hérö̈ne par les femmes doit donc être considérée dans un champ plus vaste - celui de la déviance des femmes en général et du sort qui leur est fait, deux approches qui permettent de mieux comprendre la condition des femmes.

\section{BIBLIOGRAPHIE}

ARNON, I., KLEINMAN, M.H., KISSIN, B. (1974), «Psychological differentiation in heroin addicts", International Journal of the Addictions, 9, (1), pp. 151-159.

BAKAL, D.A., ROOTMAN, I. and CAMPBELL, D. (1976), «Drug use in rural Alberta : 1976, report», Non-Medical Use of Drugs Directorate, Ottawa : Health and Welfare Canada.

BALDINGER, R., CAPEL, W.C., GOLDSMITH, B.M., STEWART, G.T. (1972), «Pot smokers, junkies and squares: A comparative study of female values», International Journal of the Addictions, 7 (1), pp. 153-166.

BARRY, K. (1979), Female Sexual Slavery, Englewood Cliffs, N.J. : Prentice-Hall.

BECKER, H. (1963), Outsiders: Studies in the sociology of deviance, New York: The Free Press of Glencoe.

BELL, D.S., CHAMPION, R.A. (1976), Monitoring drug use in New South Wales, Part 3, Division of Health Service Research, Health Commission of New South Wales, Sydney, Australia.

BERTHIAUME, M. (1984), Revue des documents écrits sur les professeurs des universités, miméo.

BETH ISRAEL MEDICAL CENTER METHADONE MAINTENANCE TREATMENT PROGRAM, (1987), Annual Report.

BINION, V. (1982), «Sex Differences in Socialization and Family Dynamics of Female and Male Heroin Users», Journal of Social Issues, 38, (2), pp. 43-57. 
BLACK, (1976), The Behavior of Law, New York : Academic Press Inc.

BLACKFORD, L. ST-CLAIR (1977), Summary report - Surveys of student drug use, San Mateo County, California : Alcohol - Amuhetamines - Barbiturates - Heroin, LSD, Marijuana, Tobacco, San Mateo County, California: Department of Public Health and Welfare.

BROWMILLER, S. (1976), Le viol, Paris: Stock (1975).

BROWN, B.S., GAUVEY, F.K., MEYERS, M.B. et STARK, S.D. (1971), "In their own words : Addicts reasons for initiating and withdrawing from heroin», International Journal of the Addictions, 6, (4), pp. 635-645.

BUREAU DES DROGUES DANGEREUSES (1985), Statistiques concernant les stupéfiants, les drogues contrôlées et les drogues d'usage restreint, Direction générale de la protection de la santé, Santé et Bien-être social, Canada.

CAPLAN, R.D. (1979), «Social support, person-environment fit, and coping", in L.A. Ferman and J.P. Gordus (eds.), Mental health and the economy, Kalamazoo, Michigan : W.E. Upjohn Institute for Employment Research.

CARDINAL, N., «La femme et la toxicomanie», in Bergeret, J. et collaborateurs (eds.) (1984), Précis des toxicomanies, Montréal : Masson et Les Presses de l'Université de Montréal, pp. 201-206.

CASSEL, J. (1976), "The contribution of the social environment to host resistance», American Journal of Epidemiology, 104, pp. 107-123.

CHEIN, I., GERARD, D., LEE, R., ROSENFELD, E. (1964), The Road to $H$. New York: Basic Books.

COBB, S. (1976), «Social support as a moderator of life stress», Psychosomatic Medicine, 38, pp. 300-314.

COLTON, M.E. (1980), A comparison of heroin-addicted and non-addicted mothers: their attitudes, beliefs and parenting experiences, NIDA, Service Research Report, Heroin-addicted parents and their children : two reports.

COVINGTON, J. (1985), «Gender differences in criminality among heroin users», Journal of Research in Crime and Delinquency, 22 (4), pp. 329-353.

D'EAUBONNE, F. (1974), Le féminisme ou la mort, Paris : Payot.

DSM III, MANUEL DIAGNOSTIQUE ET STATISTIQUE DES TROUBLES MENTAUX (1983), Traduction française placée sous la direction de P. Pichot, J.D., Gelfi.

DATABASE DIRECTORY, Ncw York: Knowledge Industry Pubiications, numero consulté : Spring 1986.

DATESMAN, S.K. (1980), Criminality of female heroin users, thèse de doctorat, University of Delaware.

DELPHY, C. (1975), «Pour un féminisme matérialiste», L'Arc, no. 61: Simone de Beauvoir et la lutte des femmes.

DEREN, S. (1986), «Children of substance abusers : A review of the literature», Journal of Substance Abuse Treatment, 3, pp. 77-94.

DESCARRIES-BÉLANGER, F; ROY, S. (1988), Le mouvement des femmes et ses courants de pensée : essai de typologie, Institut canadien de recherches sur les femmes, Ottawa. 
DHAVERNAS, M.J. (1978), «La délinquance des femmes», Questions féministes, no. 4, novembre, pp. 55-84.

DIRECTORY OF ONLINE DATABASES, New York: Cuadra Elsevier, numéro consulté : $8,(3)$, July 1987 .

DWORKIN, A. (1974), Women Hating, New York: Dutton.

FERRENCE, R.G. et WHITEHEAD, P.C. (1980), «Sex differences in psychoactive drug use", in Kalant, O., Alcohol and drug problems in women. Research Advances in Alcohol and Drug Problems, 5, pp. 125-201.

FREELAND, J.B., CAMPBELL, R.S. (1973), «The social context of first marijuana use", International Journal of the Addictions, 8, pp. 317-324.

FRENCH, M. (1985), Beyond Power: On Women, Men and Morals, New York : Summit Books.

GORE, S. (1978), «The effect of social support in moderating the health consequences of unemployment», Journal of Health and Social Behavior, 19, pp. 157-165.

GOSSOP, M. (1976), "Drug dependence and self-esteem», International Journal of the Addictions, $1 /,(5)$, pp. 741-745.

GRIFFIN, S. (1981), Pornography and Silence : Culture's Revenge Against Women, New York : Harper.

GUILLAUMIN, C. (1978), «Pratique du pouvoir et idée de nature : 1. L'appropriation des femmes", Questions féministes, no. 2, février, pp. 5-30.

GUINAN, E., HARDY, A. (1987), «Epidemiology of AIDS in women in the United States - 1981 through 1986», The Journal of American Medical Association, 257, (15), pp. 2039-2042.

HARDING, W.H., ZINBERG, N.E., STELMACK, S.M., BARRY, M. (1980), "Formerly-addicted now controlled opiate users», International Journal of the Addictions, $15,(1)$, pp. 47-60.

HOUSE, J.S. (1980), Work stress and social support, Reeding, MA: AddisonWesley.HOWARD, J., BORGES, P. (1970), «Needle sharing in the haight; social and psychological functions», Journal of Health and Social Behaviour, 11, pp. 220 230 .

HULSMAN, L. (1970), «Le choix de la sanction pénale. Revue de science criminelle et de droit pénal comparé, no. 3, pp. 497-545.

INCIARDI, J., POTTIEGER, A., FAUPEL, C. (1982), «Black women, heroin and crime : Some empirical notes», Journal of Drug /ssues, $/ 2$ (3), pp. 241-250.

KAHN, R.L., ANTONUCCI, T.C. (1980), «Convoys over the life course : Attachment, roles and social support», pp. 153-286, in P.B. Baltes and O.G. Brim (eds.), Lifespan development and behavior (vol. 3), New York : Academic Press.

KELLER, M. (1972), «The oddities of alcoholics», Quarterly Journal of Studies on Alcohol, 33, pp. 1147-1148.

LEVENTHAL, G. (1983), "Body image of drug and alcohol abusers», International Journal of the Addictions, 18 (6), pp. 791-804.

MANDON, D. (1984), «Point de vue anthropologique sur la drogue», in Bergeret, J., Leblanc, J. et collaborateurs (eds.), Précis des toxicomanies, Montréal : Masson et Les Presses de l'Université de Montréal, pp. 165-174. 
MATHIEU, N.C. (1985), «Présentation : Femmes, matières à penser... et à reproduire», L'assaisonnement des femmes, Cahiers de l'Homme, Paris: Ecole des Hautes Etudes en Sciences Sociales, pp. 5-9.

MATZA, D. (1964), Delinquency and drift, New York: Wiley.MILLER, B.A. (1980), «Drug abuse and criminality among women in detention», in Griffiths, Curt T. et Nance, Margit (eds.), Female offender, Simon Fraser University CRIMINOLOGY Research Centre, pp. 271-288.

NATIONAL INSTITUTE ON DRUG ABUSE (1980), Client Oriented Data Acquisition Process (CODAP), National Management Hankbook, Washington, D.C.: U.S. Government Printing Office.

NELKEN, D. (1973), Méthadone maintenu: A Technological Fix, New York: George Braziller.

PINNEAU, S.R. (1976), «Effects of social support on psychological and physiological strain", Dissertation Abstracts International, University of Michigan.

REED, B.G. (1985), «Drug misuse and dependency in women: The meaning and implications of being considered a special population or minority group», International Journal of the Addictions, 20 (1), pp. 13-62.

ROBERT, P. (1973), «La sociologie entre une criminologie de passage à l'acte et une criminologie de la réaction sociale», L'Année sociologique, 3ème série, vol. 24, pp. 441-504.

ROSENBAUM, M. (1979), «Becoming addicted: the woman addict», Contemporary Drug Problems, Summer, pp. 141-167.

ROSENBAUM, M. (1982), "Getting on methadone: The experience of the woman addict", Contemporary Drug Problems, Spring, pp. 113-143.

SANCHEZ, J.E., JOHNSON, B.D. (1987), «Women and the drugs-crime connection», Journal of Psychoactive Drugs, 19, pp. 205-216.

SCHUR, E. (1984), Labeling Women Deviant - Gender, Stigma and Social Control, New York : Random House.

SMART, R., FEJER, D., SMITH, D., WHITE, J. (1975), Trends in drug use among Metropolitan Toronto High School Students: 1968-1974, Toronto: Addiction Research Foundation.

SMART, R.G., GOODSTADT, M.S. and SONE, I.J. (1977), Alcohol and drug use among Ontario students in 1977, Toronto: Addiction Research Foundation.

SONTAG, S. (1979), La maladie comme métaphore, Paris : Seuil.

SUFFET, F., BROTMAN, R. (1976), «Female Drug Use: Some observations». International Journal of the Addictions, $11,(1), \mathrm{pp} .19-33$.

SUTKER, P.B., ARCHER, R.P. et ANAIR, A.N. (1980), «Psychopathology of drug abusers: sex and ethic considerations», International Journal of the Addictions, 15 , (4), pp. 605-613.

SZASZ, T. (1976), Les rituels de la drogue, Paris : Payot.

TAYLOR, I., WALTON, P., YOUNG, J. (1973), The new criminology, London: Routledge et Kegan Paul.

TUCKER, B. (1982), «Social support and coping: Applications for the study of female drug abuse», Journal of Social lssues, 38, (2), pp. 117-137. 
ZINBERG, N.E., JACOBSON, R.C., HARDING, W.M. (1975), «Social sanctions and rituals as a basis of drug abuse prevention", American Journal of Drug and Alcohol abuse, 2, pp. 165-182.

ZINBERG, N.E., JACOBSON, R.C. (1976), «The natural history of chipping», American Journal of Psychiatry, 133, pp. 37-40. 